\title{
Pancreas divisum and recurrent pancreatitis
}

\author{
RICHARD ELKIN, D.O. \\ Lancaster, Pennsylvania \\ JOHN C. CHIESA, D.O. \\ Stratford, New Jersey
}

attacks of acute pancreatitis in patients with no history of alcohol abuse or other biliary tract disease remains obscure. With the widespread use of endoscopic retrograde cholangiopancreatography, an anatomic anomaly known as pancreas divisum has been identified as a potential etiology for idiopathic acute pancreatitis. The anatomic anomaly itself arises from a failure of fusion between the ventral and the dorsal pancreatic ducts during embryologic development. Inadequate drainage by the main duct via the accessory papilla in the adult results in autodigestion, i.e., acute pancreatitis. This anatomic variant is seen in asymptomatic individuals as well, and thus raises questions of its clinical significance. In the case reported, there was recurrent idiopathic acute pancreatitis in which pancreas divisum was found. A discussion covers the current diagnosis and management.

Pancreas divisum (PD) as a potential etiology for recurrent pancreatitis is not universally accepted throughout the medical community. The case presentation in this article illustrates the association between this anatomic anomaly and recurrent acute pancreatitis. An explanation of pancreas divisum and current concepts in its diagnosis and management are presented.

\section{Report of case}

A 22-year-old Caucasian woman was seen in the emergency ward at Kennedy Memorial Hospital complaining of severe, sharp epigastric pain. The patient stated that the pain had been present since she had awakened that morning and that it radiated directly through her back. Laboratory studies revealed that the serum amylase level was elevated to twice the normal value. The serum lipase level also was markedly elevated. A diagnosis of acute pancreatitis was made, and a further history was obtained.

Interview of the patient disclosed that she had experi- enced three prior bouts of acute pancreatitis. Her first episode occurred at age 8 , and her most recent episode occurred 3 years prior to this admission, while she was in her third trimester of pregnancy. She denied ethanol abuse and smoking. She was taking no medications. Cholecystectomy had been performed on the recommendation of a surgical consultant 2 years earlier. A normal intraoperative cholangiogram had been obtained at that time, and pathologic diagnosis of the surgical specimen had confirmed a normal gallbladder free of stones.

Physical examination at the time of admission was unremarkable, with the exception of abdominal tenderness and slightly diminished bowel sounds. An obstruction radiographic series revealed a reactive gas pattern and no evidence of abdominal calcifications. The patient was put on a "nothing by mouth" regimen, and parenteral fluids and analgesics were administered. Her pain subsided over the following 36 hours, and a clear liquid diet was started. The amylase and lipase values returned to normal; a normal lipid profile also was obtained. On the fifth hospital day, the patient was free of abdominal pain and was tolerating a regular diet. Endoscopic retrograde cholangiopancreatography (ERCP) was performed, and the pancreatogram was noted to be consistent with pancreas divisum (Fig. 1).

The patient was discharged the following day pain free. No specific therapy was given at that time, and the patient was subsequently lost to follow-up.

\section{Discussion}

Pancreas divisum, an anatomic anomaly, results from the failure of fusion of the dorsal and ventral elements of the pancreas during the second month of gestation. By the seventh week of gestation, the ventral and dorsal pancreatic primordia usually are brought into direct opposition and fuse. Fusion of these elements is accompanied by anastomosis of the ventral duct, with the dorsal duct forming the main pancreatic duct (the duct of Wirsung). This duct drains the entire gland via the major papilla of Vater. The remaining proximal segment of the dorsal pancreas becomes the accessory duct (the duct of Santorini), and it drains via the accessory or minor papilla. ${ }^{1}$ Because fusion fails to occur in PD, the ducts do not anastomose, and the major drainage system of the gland consists of the duct of Santorini via the accessory papilla, while the small ventral element is drained by the major papilla of Vater (Fig. 2). 


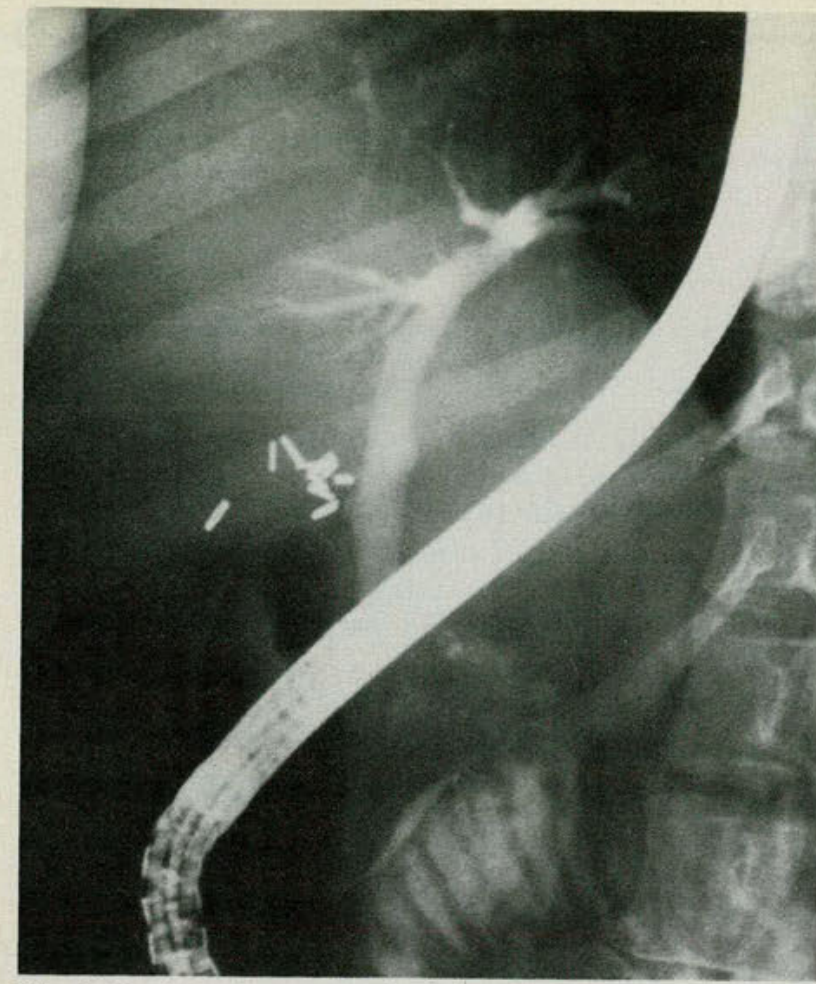

Fig. 1. Endoscopic retrograde cholangiopancreatography demonstrates filling of the common bile duct and surgical clips from prior cholecystectomy, along with filling of the ventral pancreatic duct (Wirsung). Note terminal arborization and acinarization of contrast.

Recently, the association between recurrent pancreatitis and $\mathrm{PD}$ has received much attention in the literature. The question of whether there is true etiologic association or a coincidental finding continues to be argued. The reason for the controversy stems from the fact that $\mathrm{PD}$ is a frequent congenital anomaly; it is present in up to 14 percent of cases in necropsy series and up to 5.8 percent of patients undergoing ERCP. ${ }^{2}$ It was noted, however, that among patients with unexplained recurrent pancreatitis, the incidence of PD was as high as 25.6 percent. ${ }^{2}$ Mitchell and associates, ${ }^{3}$ observing that the incidence of pancreatitis in a group with nonfusion to be similar to that found in patients with normal duct systems, disputed the association between acute pancreatitis and the unfused pancreatic duct system. Despite this finding, it now appears likely that at least in some patients PD is responsible for not only acute pancreatitis and recurrent bouts of acute pancreatitis, but for chronic pancreatitis as well. ${ }^{4}$

The current theory explaining why a minority of patients with PD develop pancreatitis is that there is relative stenosis of the accessory papilla orifice. In patients with this anatomic anomaly, the accessory papilla serves as the major outflow tract for pancreatic secretions. Warshaw and coworkers ${ }^{5}$ demonstrated pinpoint stenosis $(0.75 \mathrm{~mm}$. or less) in a high proportion of patients with acute pancreatitis and PD.

In the same study, they found that relief of stenosis by accessory papillotomy was highly effective in 16 of 18 patients. A fair response to therapy was noted in one patient while another had a poor response to papillotomy. Although sphincteroplasty was greatly effective in treating recurrent pancreatitis when the gland was histologically normal, Warshaw's group 5 emphasizes the need for ductal drainage with Peustow pancreaticojejunostomy or partial resection once chronic pancreatitis and fibrosis is established. Because of discouraging results with accessory papilla sphincteroplasty, Traverso and coauthors ${ }^{6}$ question PD as the etiology of recurrent pancreatitis as well as the utility of this type of surgery. Apparently, strict criteria for patient selection are necessary, and preoperative testing for accessory papilla stenosis would identify patients whose symptoms are caused by PD. ${ }^{5}$ Such a test was recently described by Warshaw and his coworkers at Massachusetts General Hospital. ${ }^{7}$ In that study, the pancreatic and bile duct diameters were measured using ultrasonography before and after stimulation with intravenous secretin. (The test is based on the concept that ducts will dilate if the outflow tract through the papilla is functionally inadequate to follow unimpeded emptying during maximal secretion. ${ }^{8}$ ) Although it seldom caused bile duct dilatation, secretin did cause a measurable increase in pancreatic duct diameter in 83 percent of symtomatic patients with ampullary (Oddi) stenosis and in 72 percent of symptomatic patients with accessory papilla stenosis (PD). ${ }^{7}$ Warshaw and associates $^{7}$ were able to demonstrate that this response was abolished after surgical sphincteroplasty, which indicates further that pancreatic duct dilatation after secretin injection is a function of resistance to outflow at the sphincter. According to these authors, "A positive test result was associated with a 90 percent success rate in preventing recurrent pancreatitis and ameliorating pain."

Pancreatic enzyme therapy also has been used to treat PD. Of 6 PD patients who received high doses of pancreatic extract, 3 had marked pain relief. ${ }^{9}$ Pharmacologic suppression of pancreatic exocrine secretion has been demonstrated in patients with chronic pancreatitis and in healthy subjects. ${ }^{10}$ This has been achieved by increasing the intraluminal concentration of pancreatic enzymes with orally administered exogenous enzymes. Further study will be necessary for definitive evaluation of the effect of enzyme therapy in patients with PD. 


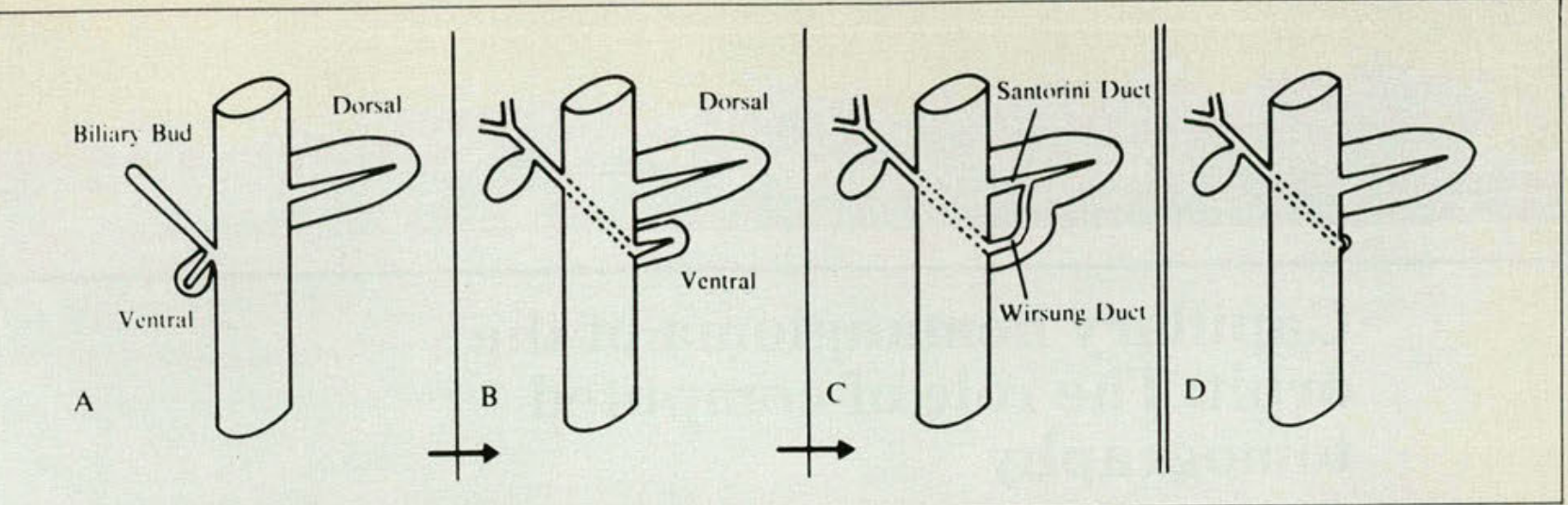

Fig. 2. Schematic representation of the embryologic development of the human pancreas. The normal adult arrangement is depicted in stage C. Pancreas divisum is shown in stages B and D (modified from Cotton ${ }^{2}$ ).

\section{Summary}

Pancreas divisum represents a developmental failure of the ducts of the dorsal and ventral pancreas to fuse during the second month of gestation. Some investigators refute the association between this anatomic anomaly and acute pancreatitis, while others feel strongly about the association. Continued investigation with ERCP coupled with a provocative test, such as the one mentioned in this article, may lead not only to a better understanding of pancreas divisum but to its clinical management as well.

1. Ermak, T.H., Grendell, J.H., and Brandborg, L.L.: The pancreas. Anatomy, embryology and developmental anomalies. In Gastrointestinal disease: Pathophysiology, diagnosis, management, edited by M.H. Sleisenger and J.S. Fordtran. Ed. 3. W.B. Saunders Co., Philadelphia, 1983 , p. 1415.

2. Cotton, P.B.: Congenital anomaly of pancreas divisum as a cause of obstructive pain and pancreatitis. Gut 21:105-14, Feb 80

3. Mitchell, C.J., et al.: Clinical relevance of an unfused pancreatic duct system. Gut 20:1066-71, Dec 79

4. Marshall, J.B., Eckhauser, M.L.: Pancreas divisum. A cause of chronic relapsing pancreatitis. Digest Dis Sci 30:582-7, Jun 85

5. Warshaw, A.L., Richter, J.M., and Schapiro, R.H.: The cause and treatment of pancreatitis associated with pancreas divisum. Ann Surg 198:443-52, Oct 83

6. Traverso, L.W., et al.: Pancreas divisum. The role of pancreatic ductal drainage. Surg Gastroenterol 1:116, 1982

7. Warshaw, A.L., et al.: Objective evaluation of ampullary stenosis with ultrasonography and pancreatic stimulation. Am J Surg 149:65-72, Jan 85

8. Simeone, J.F., et al.: The bile ducts after a fatty meal. Further sonographic observations. Radiology 154:763-8, Mar 85

9. Toskes, P.P: Recurrent acute pancreatitis. Hosp Prac 20:85-92, 15 Jul 85

10. Slaff, J., et al.: Protease. Specific suppression of pancreatic exocrine secretion. Gastroenterology 87:44-52, Jul 84

At the time this paper was written, Dr. Elkin was a fellow in gastroenterology at the University of Medicine and Dentistry of New Jersey, School of Osteopathic Medicine, Stratford, New Jersey. He is now in the private practice of gastroenterology in Lancaster, Pennsylvania. Dr. Chiesa is an associate professor of medicine and head of Division of Gastroenterology at UMDMJSOM, and head, Division of Gastroenterology, Kennedy Memorial Hospitals/University Medical Center, Stratford Division.

Dr. Elkin, 1253 Wheatland Avenue, Lancaster, Pennsylvania 17603. 